\title{
Transnasal Endoscopy is Preferred by Transoral Endoscopy Experienced Patients
}

\author{
Abdurrahman Kadayifci ${ }^{1}$, Mustafa Atar ${ }^{2}$, Serap Parlar ${ }^{3}$, Ayhan Balkan ${ }^{1}$, Irfan Koruk ${ }^{1}$, Mehmet Koruk ${ }^{1}$
}

1) Division of

Gastroenterology, Faculty of Medicine, University of

Gaziantep, Gaziantep, Turkey

2) Division of

Gastroenterology,

Massachusetts General

Hospital, Harvard Medical

School, Boston, MA

3) Department of Internal

Medicine Nursing, Faculty of

Health Science, University of

Gaziantep

Gaziantep, Turkey

Address for correspondence:

Dr. Abdurrahman Kadayifci

3-H GI Associates, Zero

Emerson Place, Blossom st.

Massachusetts General

Hospital, Boston, 02114

USA

akadayifci@mgh.harvard.edu kadayifci@hotmail.com

Received: 16.01.2014

Accepted: 25.02.2014

\section{ABSTRACT}

Background \& Aims: Both unsedated transoral endoscopy (TOE) and sedated TOE have some drawbacks in clinical practice. Unsedated transnasal endoscopy (TNE) has been suggested as an alternative to both methods. This study aimed to determine the advantages of TNE in patients who have previously undergone unsedated conventional TOE.

Methods: Patients who had received an unsedated TOE in the last 12 months and were scheduled for a second upper endoscopy were included. They were randomized to undergo either unsedated TOE, using a standard endoscope, or unsedated TNE, using an ultrathin endoscope. Post-procedure, patients were asked to complete a questionnaire to assess pain, discomfort and acceptability of the procedure, and to compare the current procedure with their previous unsedated TOE. Endoscope insertion rate, procedure duration, and side-effects were recorded.

Results: Each group included 50 patients. With the exception of nasal pain, the tolerability and acceptance were significantly greater in the unsedated TNE group. Significantly more TNE patients (82\%) found the current endoscopic procedure to be better than their previous TOE when compared with patients who had received a second TOE (12\%). A repeat procedure was significantly more acceptable for TNE patients when compared to the TOE group ( $68 \%$ vs.16\%). The duration of endoscopy was significantly shorter in TOE than in TNE $(\mathrm{p}<0.05)$. Endoscope insertion failed in $4 \%$ and mild epistaxis was observed in $4 \%$ of TNE patients. Conclusion: Unsedated TNE was better tolerated in endoscopy experienced patients when compared with unsedated TOE. The majority of patients found TNE more acceptable and preferable to TOE, suggesting that TNE should become a more common practice in clinics when applicable.

Key words: Transnasal endoscopy - ultrathin endoscope - unsedated endoscopy - tolerability.

\section{INTRODUCTION}

Upper gastrointestinal (GI) endoscopy is the most common procedure in GI clinical practice. It can be performed both sedated and unsedated according to the patients', endoscopists' or institutions' preferences. Both methods are common in GI practice, but have significant drawbacks. Unsedated transoral endoscopy (TOE) can significantly increase patient's discomfort and decrease the tolerability of the procedure. It may also decrease the quality of the examination, as a result of the patients' gag reflex and general discomfort $[1,2]$. Sedated endoscopy is more comfortable and acceptable for the patient than unsedated TOE. However, sedation increases the risk of any procedure, especially in patients with cardiovascular problems and in older populations [3-5]. In addition, sedated TOE requires venous access, a recovery room, and extra care which generally increase the cost of the procedure [6].

Unsedated transnasal endoscopy (TNE) with an ultrathin endoscope (outer diameter, OD, less than $6 \mathrm{~mm}$ ) has been introduced as an alternative method to both unsedated and sedated upper GI endoscopy [7-10]. Previous studies have shown that this method can resolve the major disadvantages associated with both sedated and unsedated TOE, providing a more comfortable endoscopy without intravenous sedation $[11,12]$. Most clinical studies comparing TNE with TOE have been done in patients with no previous GI endoscopic procedure. However, the evaluation of TNE by patients who have had a previous TOE experience may provide more useful information regarding the tolerability and acceptability of this method as well as the utility of the procedure for diagnostic purposes. Such a study design has not been done before. The 
primary objective of the current study was to determine the tolerability and acceptance of TNE in patients who had a previous experience with unsedated conventional TOE. The secondary objective was to evaluate the drawbacks of TNE.

\section{METHODS}

This prospective, randomized clinical study was performed in a high volume endoscopy center of the Gaziantep University Hospital between 2010 and 2012. The study group consisted of patients who had previously received an unsedated TOE in the last 12 months and were scheduled for a second upper GI endoscopy. A study co-investigator checked the medical records of patients scheduled for upper endoscopic procedures daily during the study period and contacted all potential candidates. Patients with a history of nasal trauma or surgery, coagulopathy, the suspicion of an active GI bleeding, undergoing a planned or likely therapeutic endoscopy, the inability to complete the study survey, or scheduled for a sedated endoscopy for any reason were excluded from the study. The techniques, details and differences of both procedures were explained to all eligible patients by a study co-investigator, and patients were enrolled only if they accepted the study protocol (approved by the local Ethical Committee) and gave their consent.

All eligible patients were randomly allocated with a 1:1 ratio to either the unsedated TOE group or the unsedated TNE group. Patients in the TOE group received only local pharyngeal anesthesia with lidocaine spray prior to the procedure. The endoscopy was done using a standard endoscope (OD: 9.3 or 9.8 mm, Fujinon EG-450 or Olympus GIF-Q160). Patients in the TNE group first received lidocaine spray to the nostrils and pharynx. A nasal pretreatment catheter (Fujinon 14 or 16 Fr diameter and 90 mm length, see Fig.1) coated with lidocaine gel was then inserted gently into the nostril which the patient could breathe more easily. After 3 minutes, the catheter was removed and TNE was performed using an ultrathin endoscope (OD: $5.9 \mathrm{~mm}$, Fujinon EG$530 \mathrm{~N})$. Three senior endoscopists, who had experience in

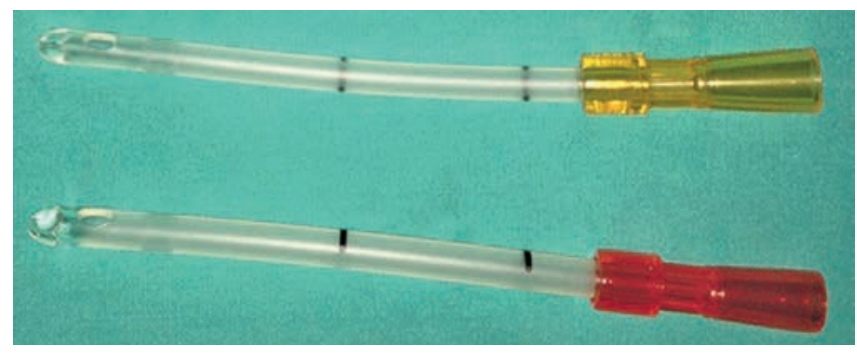

Fig. 1. Pretreatment delivery catheter for transnasal endoscopy (Fujinon, Fujilm, Japan)

TNE, performed all procedures. If the transnasal insertion failed, the procedure was completed by oral route with the ultrathin endoscope. Biopsies were taken only where clinically indicated. The working channel of ultrathin endoscopes is of $2 \mathrm{~mm}$. If a biopsy sample was needed, it was obtained using a $1.8 \mathrm{~mm}$ diameter biopsy forceps made specifically for ultrathin endoscopes. Blood pressure, pulse rate, and oxygen saturation were constantly monitored during all procedures. Heart rate over 100 beats per minute (bpm) was considered tachycardia, and a drop in $\mathrm{SaO} 2$ below $90 \%$ was considered hypoxemia during the procedure. Supplemental oxygen was given by nasal cannula if hypoxemia lasted more than 30 seconds. The duration of procedure and the occurrence of any side effects were recorded at the conclusion of the procedure in all patients.

After the procedure, all patients were asked to complete a questionnaire regarding their pain and discomfort during the procedure and the tolerability of the endoscopy. A validated visual analog scale (VAS) based on a scale of 1 to 10 ( 1 =Minimal, $5=$ Moderate, $10=$ Severe symptom) was used for evaluation of the procedures (Table I). A senior nurse explained the questionnaire in detail prior to all patients' completion of the survey. Patients were also asked to compare the current procedure with the one they had previously received within the last 12 months. As a final question, patients were asked their preference for any potential endoscopic procedures in the future.

Table I. The queries included in the questionnaire form.

\begin{tabular}{ll}
\hline Query & Evaluation \\
\hline Please describe your apprehension, anxiety and concern before the procedure & VAS: 0-10 \\
Please describe the pain you felt inside your nose during the procedure & VAS: 0-10 \\
Please describe the pain you felt in your throat during the procedure & VAS: 0-10 \\
Please describe the feeling of retching and breathlessness during the procedure & VAS: 0-10 \\
Please describe the discomfort and pain in your abdomen during the procedure & VAS: 0-10 \\
Please describe how tolerable is this procedure & VAS: 0-10 \\
Please describe the overall distress and difficulty of the procedure & VAS: 0-10 \\
If you compare difficulty and tolerability of the current procedure with your previous one, & a) Both were the same \\
If it is required to repeat this procedure in future, which of them do you prefer? & b) The last one was better \\
\end{tabular}

$0=$ No, $1=$ Minimal, $5=$ Moderate, $10=$ Severe symptom 
The estimated sample size was calculated to detect a 2-point difference in mean VAS scores $( \pm 3$ standard deviation, SD) between groups. To detect this difference with $90 \%$ power at a $5 \%$ significance level, 48 patients were required in each study arm. This sample size also provided enough power to detect the differences in other data between groups. All data is expressed as mean $\pm \mathrm{SD}$. Comparisons between groups were done using independent Student $t$ test. Categorical variables were tested with Chi Square test and Fisher's exact test. A twotailed $\mathrm{p}$ value less than 0.05 was considered significant. SPSS 15.0 package program (2006 SPSS Inc., Chicago, IL) was used for statistical analysis.

\section{RESULTS}

During the study, outpatient patients who were scheduled for an upper endoscopy were screened by study co-investigators for eligibility and 428 patients were detected. The study was conducted with 100 patients in total who approved the protocol and gave their consent. After randomization, 50 patients were included in each study group. Demographic and clinical characteristics were similar between groups (Table II). The procedures were completed successfully in all 50 patients within the TOE group (100\%) and 48 of the patients in the TNE group (96\%). The nasal insertion failed in 2 TNE patients because of narrow nasal passages ( 1 patient) and severe nasal pain (1 patient). The procedure was performed by transoral insertion in these two patients and no nasal complications or significant symptoms occurred. Endoscopic examination was completed in the remaining TNE patients as described in the methods. Patients, whose nasal insertion failed, were excluded

Table II. Demographic and clinical data of the patients.

\begin{tabular}{|c|c|c|c|}
\hline Characteristics & TOE & TNE & $\mathrm{p}$ \\
\hline Patients (n) & 50 & 50 & \\
\hline Age (mean $\pm \mathrm{SD}$, years) & $47.1 \pm 11.3$ & $45.2 \pm 12.9$ & $0.43^{\mathrm{a}}$ \\
\hline Gender (male/female) & $23 / 27$ & $28 / 22$ & $0.42^{\mathrm{b}}$ \\
\hline $\begin{array}{l}\text { Previous endoscopy } \\
\text { (median, weeks) }\end{array}$ & 21 & 18 & $0.68^{\mathrm{b}}$ \\
\hline \multicolumn{4}{|c|}{ Indication for current upper endoscopy } \\
\hline Dyspeptic symptoms & 15 & 13 & $0.82^{\mathrm{b}}$ \\
\hline Epigastric pain & 11 & 10 & $1^{\mathrm{b}}$ \\
\hline Pyrosis, regurgitation & 8 & 6 & $0.77^{\mathrm{b}}$ \\
\hline Anemia & 3 & 7 & $0.31^{\mathrm{b}}$ \\
\hline Screening & 8 & 11 & $0.61^{\mathrm{b}}$ \\
\hline Others & 5 & 3 & $0.71^{c}$ \\
\hline Successful completion & $50(100 \%)$ & $48(96 \%)$ & $0.49^{c}$ \\
\hline $\begin{array}{l}\text { Duration of endoscopy } \\
\text { (mean } \pm \mathrm{SD} \text {, minute) }\end{array}$ & $6.1 \pm 4.2$ & $7.7 \pm 3.3$ & $0.03^{\mathrm{a}}$ \\
\hline Biopsy during endoscopy (n) & 22 & 17 & $0.50^{\mathrm{b}}$ \\
\hline Complications (n) & 3 & 2 & $1^{\mathrm{c}}$ \\
\hline Epistaxis & 0 & 2 & $0.49^{c}$ \\
\hline Tachycardia & 2 & 0 & $0.49^{\mathrm{c}}$ \\
\hline Hypoxemia & 1 & 0 & $1^{\mathrm{c}}$ \\
\hline
\end{tabular}

SD: Standard Deviation ${ }^{\mathrm{a}}$ :Unpaired Student $t$ test, ${ }^{\mathrm{b}}$ :Chi-Square test with Yates corrected, ${ }^{c}$ :Fisher exact test; TOE: transoral endoscopy; TNE: transnasal endoscopy. from subsequent analysis. The duration of the procedure was significantly shorter (by $\sim 1.5$ minutes) in TOE patients when compared with TNE patients (Table II).

Apprehension, anxiety, and concern about the endoscopy prior to the procedure were similar in both groups (Table III). Mean VAS scores for abdominal discomfort and pain during the procedure were also not significantly different between two groups. Mean nasal pain score was significantly higher in the TNE group than in TOE patients. However, pain in throat, retching and feeling of breathlessness, overall distress and difficulty of the procedure were reported significantly higher in the TOE group when compared to the TNE patients (Table III).

A majority of patients (82\%) found TNE better in terms of tolerability and discomfort than their previous TOE procedure (Table IV). Only $6 \%$ of patients reported that their TOE experience was better than TNE, while $12 \%$ evaluated both as equal with regard to tolerability. Nevertheless, most patients (78\%) in the TOE group did not report any difference in discomfort with their previous TOE procedure. Only $12 \%$ patients found the second TOE to be more tolerable and 10\% considered their first TOE to be better. For a possible future endoscopic procedure, $84 \%$ of patients in the TOE group asked for an alternative method while only $32 \%$ of patients in the TNE group sought for an alternative method of treatment (Table IV).

Both methods were well tolerated and no significant adverse effects were detected over the course of the study. Mild epistaxis occurred in 2 patients in the TNE group but stopped spontaneously without requiring any intervention. Tachycardia and hypoxemia were detected in 3 patients from the TOE group, but no significant cardiorespiratory event was detected.

Table III. VAS results (mean $\pm \mathrm{SD}$ ) obtained from questionnaire.

\begin{tabular}{llll}
\hline Queries & TOE & TNE & $\mathrm{p}^{\mathrm{a}}$ \\
\hline $\begin{array}{l}\text { Apprehension, anxiety and concern } \\
\text { before the procedure }\end{array}$ & $3.78 \pm 3.2$ & $3.4 \pm 3.7$ & 0.58 \\
Pain inside the nose & $0.9 \pm 0.6$ & $3.7 \pm 2.3$ & 0.0001 \\
Pain in the throat & $4.1 \pm 2.8$ & $1.6 \pm 0.7$ & 0.0001 \\
Retching and breathlessness feeling & $4.8 \pm 3.4$ & $2.0 \pm 1.3$ & 0.0001 \\
Abdominal discomfort and pain & $3.6 \pm 1.6$ & $3.1 \pm 1.8$ & 0.14 \\
Tolerability & $4.5 \pm 2.4$ & $2.7 \pm 2.1$ & 0.0002 \\
Overall distress and difficulty & $4.3 \pm 2.9$ & $2.2 \pm 1.6$ & 0.0001 \\
\hline
\end{tabular}

SD: Standard Deviation ${ }^{a}$ :Unpaired Student $t$ testTOE: transoral endoscopy; TNE: transnasal endoscopy.

Table IV. Patients' comparison with previous endoscopy and preferences for future.

\begin{tabular}{|c|c|c|c|}
\hline Evaluation & TOE n, (\%) & TNE n, (\%) & $\mathrm{p}$ \\
\hline \multicolumn{4}{|c|}{$\begin{array}{l}\text { Compare difficulty and tolerability of the current procedure with the } \\
\text { previous one. }\end{array}$} \\
\hline Both were same & $39(78 \%)$ & $6(12 \%)$ & $0.0001^{a}$ \\
\hline The last one was better & $6(12 \%)$ & $41(82 \%)$ & $0.0001^{a}$ \\
\hline The first one was better & $5(10 \%)$ & $3(6 \%)$ & $0.71^{\mathrm{b}}$ \\
\hline \multicolumn{4}{|c|}{$\begin{array}{l}\text { If it is required to repeat this procedure in future; which of them do you } \\
\text { prefer? }\end{array}$} \\
\hline The same method & $8(16 \%)$ & $34(68 \%)$ & $0.0001^{a}$ \\
\hline An alternative method & $42(84 \%)$ & $16(32 \%)$ & $0.0001^{a}$ \\
\hline
\end{tabular}

${ }^{\mathrm{a}}$ :Chi-Square test with Yates corrected, ${ }^{\mathrm{b}}$ :Fisher exact testTOE: transoral endoscopy; TNE: transnasal endoscopy. 


\section{DISCUSSION}

After the development of ultrathin endoscopes, unsedated TNE has been suggested as a safe and cost effective alternative method for endoscopic examination of the upper GI tract [1114]. In the current study, different from previous studies, we compared the tolerability of TNE to TOE in patients who had received a TOE procedure in the past 12 months. We thought such an experimental design could potentially provide more comparable data, while giving patients an opportunity to evaluate and compare both endoscopic methods. While the primary aim of the study was the evaluation of TNE by patients who had previously received a TOE, we randomized patients into TNE and TOE groups to prevent a possible bias of second endoscopic procedure.

In this study, TNE was tolerated significantly better by patients than unsedated TOE. Most importantly, a significant proportion of TNE patients (82\%) reported that they tolerated the second procedure better than their previous unsedated TOE. For patients who received a second TOE, the results were significantly different than those of the TNE group and most patients (78\%) found the current endoscopy was comparable with their past experience. As a confirmation of this result, the majority of patients in TNE group made their preference for a TNE in future. These data clearly showed that TNE is more preferable than unsedated TOE in terms of patients' symptoms and tolerability. In addition, experiencing a TNE improves patients' perception of upper endoscopy and changes their preference in future. A recent study comparing TNE with standard and ultrathin unsedated TOE in geriatric patient groups could not find a superiority of TNE over TOE [15]. In another prospective study which consisted of patients undergoing periodical screening endoscopy, $68.7 \%$ of patients preferred TNE and $31.3 \%$ preferred TOE based on their experience with discomfort in previous examinations [16]. The preference of TNE was more significant in male and younger patients. The flexibility of ultrathin endoscopy was the most significant predictor of reduction in TNE-associated discomfort. These studies revealed that age and gender may affect the preference of endoscopy insertion route. We could not show any effect of age and gender for patient preference in the current study. However, further studies designed with adequate sample size are required.

Our study has also shown some drawbacks to TNE. From the patient perspective, the most significant symptom of TNE was nasal pain. However, most patients did not complain of this and tolerated the procedure well. A clinical study found that older patients are likely to be less sensitive than younger during the insertion and withdrawal of scope from the nose probably due to a reduced sensitivity of intranasal trigeminal system $[17,18]$. In another study, patients were specifically asked about pain and $55 \%$ of all patients reported that the most painful region was the nose during the procedure. However, $65 \%$ of all patients who underwent a TOE in the same study reported that the pharynx was the most painful region [19]. Pharyngeal pain was also more significant in the TOE group in our current study. These results showed that some patients may suffer from nasal pain and discomfort during the nasal insertion, but it is usually tolerable, and comparable to the pain and discomfort caused by oral insertion. We think that nasal pretreatment and application of an appropriate local anesthesia are the most critical parts of the TNE procedure and, if done correctly, can significantly reduce nasal pain. As a result, nasal discomfort is expected with TNE, just as pharyngeal pain is expected with TOE. However, the nasal cavity can be more easily locally anesthetized which should help reduce or even eliminate the pain associated with TNE.

The most significant and common complication associated with TNE was epistaxis in previous studies $[13,20]$. It was mostly reported between 1 to $5 \%$ in clinical studies and most cases were mild, self-limited (stopping within a few minutes after the procedure), and did not need any intervention. The rate of epistaxis in our current study was $4 \%$ which is comparable to the literature. Patients did not require any therapeutic intervention, and bleeding stopped spontaneously. There was no significant change in the vital signs TNE patients and the procedure was completely safe for the cardiorespiratory system.

Another disadvantage of TNE is insertion failure. In this study, the endoscope insertion failure rate was $4 \%$ and the procedure was completed in these patients via oral route without delay. In a large study, consisting of 1100 patients, the insertion failure rate of TNE was 6.1\% [21]. Different studies have reported failure rates ranging from 0 to $10 \%$ with TNE. This variability is likely due to the experience of endoscopist, scope diameter and nasal pretreatment protocol [11, 21-23]. Therefore, while insertion failure is a significant drawback of TNE, it is quite easy to switch to the oral route and this imposes no negative effect on patients. Pharyngeal topical anesthesia during pretreatment makes such a switch particularly easy. In our study, we found the duration of TNE was significantly longer than TOE by $\sim 1.5$ minutes. However, this did not appear to have a significant impact on the tolerability of the procedure.

One important drawback of ultrathin endoscopes are their narrow working channel and poor suction and air functions when compared to standard endoscopes [24]. Besides, these scopes have a limited numbers of available endoscopic accessories and are not appropriate for therapeutic procedures through the working channel and this is a true limitation of this method. Thus, TNE is mostly used for diagnostic purposes except in certain conditions which require a thinner endoscope [25]. The current study was performed on a selected patient group comprising uncomplicated cases. The patients who were planned or likely to have a therapeutic endoscopy were excluded from the study.

A key limitation to our current study was the reliance on patient memory when asking patients to compare their current procedure with their previous unsedated TOE. The study was designed with two arms to reduce the negative effect of a historical comparison. Without doubt, two consecutive recent endoscopies with TNE and TOE would be more valuable to compare both methods. However, patient recruitment for such a design based on an acceptable clinical requirement would be difficult, and the ethical aspects of doing two endoscopies in the absence of any clinical basis are also questionable. Furthermore, despite this limitation, both primary and secondary objectives of our study have been fulfilled. 


\section{CONCLUSION}

Unsedated TNE is a safe procedure with a high success rate with few complications. It is more tolerable than unsedated TOE, and patients who received a previous TOE procedure in the past year found TNE more acceptable and preferable to TOE. There are some drawbacks and limitations of the TNE, but they are manageable. Patients scheduled for an upper GI endoscopy who are eligible for TNE should be informed about the advantages and disadvantages of this method and offered the procedure as an alternative to standard unsedated TOE.

Conflicts of interest. All authors certify that there is no conflict of interest with any financial organization regarding the material presented in the manuscript.

\section{REFERENCES}

1. Thanvi BR, Munshi SK, Vijayakumar N, Taub N, Lo TC. Acceptability of oesophagogastroduodenoscopy without intravenous sedation: patients versus endoscopist's perception with special reference to older patients. Postgrad Med J 2003;79:650-651.

2. Faulx AL, Vela S, Das A, et al. The changing landscape of practice patterns regarding unsedated endoscopy and propofol use: a national Web survey. Gastrointest Endosc 2005;62:9-15.

3. Travis AC, Pievsky D, Saltzman JR. Endoscopy in the elderly. Am J Gastroenterol 2012;107:1495-1501; quiz 1494, 1502.

4. Abraham NS, Fallone CA, Mayrand S, Huang J, Wieczorek P, Barkun AN. Sedation versus no sedation in the performance of diagnostic upper gastrointestinal endoscopy: a Canadian randomized controlled cost-outcome study. Am J Gastroenterol 2004;99:1692-1699.

5. Ginzburg L, Greenwald D, Cohen J. Complications of endoscopy. Gastrointest Endosc Clin N Am 2007;17:405-432.

6. Liu H, Waxman DA, Main R, Mattke S. Utilization of anesthesia services during outpatient endoscopies and colonoscopies and associated spending in 2003-2009. JAMA 2012;307:1178-1184.

7. Frieling T, Schindler P, Kuhlbusch-Zicklam R, Heise J, Hülsdonk A, Kreysel C. Krefeld CONTRA study: conventional peroral EsophagoGastro-Duodenoscopy (EGD) vs. transnasal EGD--a prospective and randomised study with independent evaluation of conscious sedation, endoscope diameter, and access path. Z Gastroenterol 2010;48:818824.

8. Horiuchi A, Nakayama Y, Hidaka N, Ichise Y, Kajiyama M, Tanaka N. Prospective comparison between sedated high-definition oral and unsedated ultrathin transnasal esophagogastroduodenoscopy in the same subjects: pilot study. Dig Endosc 2009;21:24-28.

9. Stroppa I, Grasso E, Paoluzi OA, et al. Unsedated transnasal versus transoral sedated upper gastrointestinal endoscopy: a one-series prospective study on safety and patient acceptability. Dig Liver Dis 2008;40:767-775.

10. Cho S, Arya N, Swan K, et al. Unsedated transnasal endoscopy: a Canadian experience in daily practice. Can J Gastroenterol 2008;22:243 246.

11. Lee SY, Kawai T. Transnasal route: new approach to endoscopy. Gut Liver 2008;2:155-165.

12. Adler DG. Ultrathin transnasal endoscopy without sedation: the straight skinny. Gastrointest Endosc 2012;75:962-964.

13. Tatsumi Y, Harada A, Matsumoto T, Tani T, Nishida H. Current status and evaluation of transnasal esophagogastroduodenoscopy. Dig Endosc 2009;21:141-146.

14. Preiss C, Charton JP, Schumacher B, Neuhaus H. A randomized trial of unsedated transnasal small-caliber esophagogastroduodenoscopy (EGD) versus peroral small-caliber EGD versus conventional EGD. Endoscopy 2003;35:641-646.

15. Uchiyama K, Ishikawa T, Sakamoto N, et al. Analysis of cardiopulmonary stress during endoscopy: Is unsedated transnasal esophagogastroduodenoscopy appropriate for elderly patients? Can J Gastroenterol 2014;28:31-34.

16. Ono S, Niimi K, Fujishiro M, et al. Ultrathin endoscope flexibility can predict discomfort associated with unsedated transnasal esophagogastroduodenoscopy. World J Gastrointest Endosc 2013;5:346351.

17. Murata A, Akahoshi K, Motomura Y, et al. Prospective comparative study on the acceptability of unsedated transnasal endoscopy in younger versus older patients. J Clin Gastroenterol 2008;42:965-968.

18. Frasnelli J, Hummel T. Age-related decline of intranasal trigeminal sensitivity: is it a peripheral event? Brain Res 2003;987:201-206.

19. Watanabe $\mathrm{H}$, Watanabe $\mathrm{N}$, Ogura R, et al. A randomized prospective trial comparing unsedated endoscopy via transnasal and transoral routes using 5.5-mm video endoscopy. Dig Dis Sci 2009;54:2155-2160.

20. Maffei M, Dumonceau JM. Transnasal esogastroduodenoscopy (EGD): comparison with conventional EGD and new applications. Swiss Med Wkly 2008;138:658-664.

21. Dumortier J, Napoleon B, Hedelius F, et al. Unsedated transnasal EGD in daily practice: results with 1100 consecutive patients. Gastrointest Endosc 2003;57:198-204.

22. Trevisani L, Cifala V, Sartori S, Gilli G, Matarese G, Abbasciano V. Unsedated ultrathin upper endoscopy is better than conventional endoscopy in routine outpatient gastroenterology practice: a randomized trial. World J Gastroenterol 2007;13:906-911.

23. ASGE Technology Committee, Rodriguez SA, Banerjee S, et al. Ultrathin endoscopes. Gastrointest Endosc 2010;71:893-898.

24. Kawai T, Yamamoto K, Fukuzawa M, Sakai Y, Moriyasu F. Ultra-thin transnasal esophagogastroduodenoscopy. Nihon Rinsho 2010;68:12641267.

25. Aydinli M, Koruk I, Dag MS, Savas MC, Kadayifci A. Ultrathin endoscopy for gastrointestinal strictures. Dig Endosc 2012;24:150-153. 